\title{
L'« année-recherche ", un dispositif précieux pour la formation des internes à la recherche. L'expérience du Centre hospitalier universitaire de Lyon
}

\author{
The "Research-Year", a valuable program for residents dedicated \\ to research training. The experience of the university and public hospitals \\ of Lyon (France)
}

En France, le dispositif « année recherche » permet aux internes (étudiants en formation spécialisée de troisième cycle) en médecine, pharmacie ou odontologie, à partir de leur deuxième année d'internat, d'interrompre leur parcours médical pendant un an, pour se former « à et par la recherche », tout en conservant leur statut et rémunération d'interne. Cette année est financée par l'Assurance Maladie. Les modalités d'organisation de l' « année recherche », durant le troisième cycle, sont énoncées dans l'arrêté du 21 janvier 2016 modifiant l'arrêté du 4 octobre $2006^{[1]}$.

\section{Contexte et problématique}

L'objectif de ce dispositif est de permettre à des internes en cours de formation, qui désirent s'initier à la recherche ou approfondir leur thématique de recherche, de disposer d'une période de douze mois pour mener à bien un projet de recherche. L'intérêt de ce dispositif est multiple, tant au niveau personnel qu'institutionnel. Il s'agit tout d'abord de renforcer l'enseignement des sciences fondamentales chez les étudiants en médecine. En effet, de nombreux internes peuvent ainsi valider un master en s'appuyant sur la validation du deuxième cycle des études médicales et des épreuves classantes nationales (équivalence première année de master). Ceci crée une interface dynamique entre les facultés de médecine, de science, les hôpitaux et les laboratoires en permettant aux internes de suivre des enseignements fondamentaux, puis de participer à des activités de recherche ayant un impact potentiel en relation avec leur pratique clinique. Il en résulte un dynamisme entre les enseignants chercheurs (cliniciens ou scientifiques) visant à proposer des intitulés/programmes de master et des projets en laboratoire en accord avec les domaines d'expertise de l'université et du centre hospitalier universitaire de rattachement. Pour l'institution, la formation de professionnels de santé disposant d'un bagage scientifique solide est un atout car elle permet de disposer d'une force innovante, pouvant être intégrée dans les cursus hospitalouniversitaires. L'avènement de la médecine basée sur les preuves (evidence-based medicine) et l'hyperspécialisation de la pratique clinique exigent des connaissances théoriques et pratiques en science qui vont bien au-delà des enseignements communs des études de médecine, tant pour comprendre et analyser des études que pour initier, financer, superviser et valoriser des programmes de recherche. L'« année 
recherche » répond à cette exigence de formation et on ne peut que soutenir la tendance actuelle visant à augmenter le nombre de lauréats pouvant en bénéficier.

Le Ministère de la Santé fixe chaque année, par subdivision, le nombre d'internes susceptibles de pouvoir bénéficier du financement « année-recherche ». Une commission interrégionale sélectionne ensuite, sur dossier, les internes qui pourront bénéficier de ce financement. Les internes non retenus peuvent faire une année de recherche, mais doivent se mettre en disponibilité et trouver eux-mêmes d'autres sources de financement. La commission interrégionnale de sélection est composée des directeurs des unités de formation et de recherche de rattachement, des viceprésidents de directoire chargés de la recherche des centres hospitaliers universitaires de rattachement, des présidents des universités de rattachement et de chercheurs titulaires désignés par le délégué régional de la recherche et de la technologie au sein des organismes de recherche et sciences de la vie et de la santé. La commission évalue les dossiers soumis par les candidats et sélectionne les meilleurs dossiers en fonction de la qualité scientifique du projet, du laboratoire d'accueil, et de la motivation du candidat. Le rang de classement aux épreuves classantes nationales n'est plus un critère de sélection. L' « annéerecherche » s'effectue au cours de l'année universitaire suivant son attribution.

\section{Enquête rétrospective " année-recherche " 2010-2014}

Nous avons mené une enquête rétrospective sur cinq ans, afin d'évaluer le processus de sélection et les attentes des internes ayant postulé au financement « année-recherche » dans la subdivision de Lyon entre 2010 et 2014. Parmi les internes non retenus à l'issue du processus de sélection, nous avons évalué le pourcentage d'internes ayant néanmoins réussi à réaliser leur projet de recherche et le type de financement qu'ils ont utilisé. Nous avons envoyé un questionnaire, par message électronique, à l'ensemble des 112 internes ayant candidaté au financement « annéerecherche », dans la subdivision de Lyon durant la période concernée. Le questionnaire comportait 19 questions portant sur : le candidat (données démographiques, formation, spécialisation, projet universitaire), le projet scientifique soumis (recherche fondamentale, translationnelle ou clinique), l'obtention ou non du financement « année-recherche », la réalisation ou non du projet de recherche et le financement utilisé en cas de non attribution du financement «année-recherche». Trois relances ont été effectuées suivant l'envoi initial.

\section{Taux de réussite en fonction du diplôme d'études spécialisé d'origine}

En utilisant les données administratives relatives au financement « année-recherche » sur les cinq dernières années, nous avons pu établir que le taux de réussite, c'est-à-dire le pourcentage d'internes ayant obtenu le financement " année-recherche » est en constante augmentation depuis 2011, passant de $24 \%$ à $62 \%$ (figure 1) avec un taux moyen sur cinq ans de $41 \%$, ceci s'expliquant principalement par l'augmentation du nombre de financements disponibles à partir de 2013 ( 7 postes en 2012 contre 16 en 2013). La poursuite de cette progression en 2014 est due à un nombre moins important de candidatures (26 en 2014 contre 32 en 2013).

Les projets présentés au financement « annéerecherche » font ressortir les particularités du réseau médico-scientifique lyonnais avec une prédominance de projets intéressant les neurosciences, l'oncologie et l'immunologie.

Les candidats sont issus de la quasi totalité des formations aux diplômes d'études spécialisées (DES), à l'exception de la médecine du travail, de la médecine nucléaire, et de la radiothérapie. Les taux de succès par DES (pourcentage de projets retenus par rapport aux projets présentés par spécialité), après exclusion des filières ayant présenté un nombre très restreint de candidats (strictement inférieur à 5 , soit moins de 1 par an), sont variables. La biologie médicale présente un taux de succès de $0 \%$ puisque aucun des cinq projets n'a été retenu sur la période analysée. La spécialité avec le plus faible pourcentage de réussite $(22 \%=2 / 9)$, après la biologie 


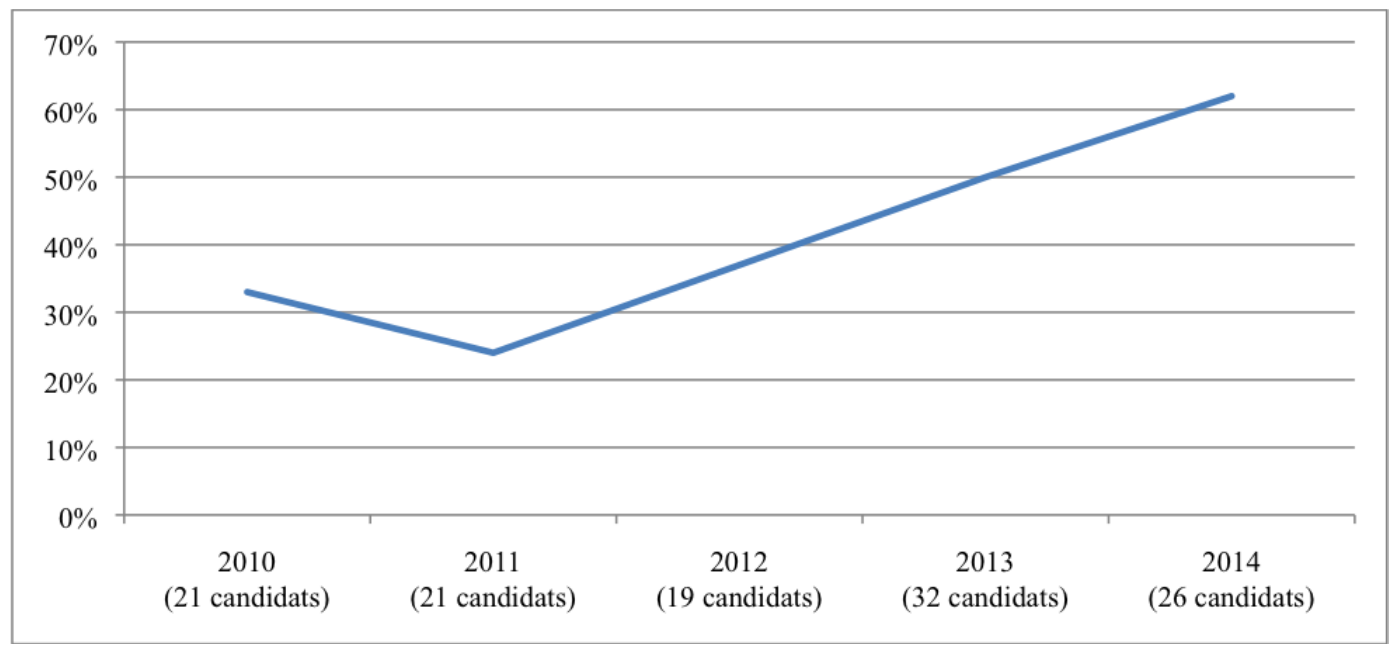

Fig. 1. Evolution du pourcentage d'internes ayant obtenu le financement «année-recherche » parmi l'ensemble des candidatures au cours des cinq dernières années. En abcisses, le nombre de candidatures par année étudiée.

médicale, est l'anesthésie réanimation. Les deux spécialités avec le plus fort taux d'acceptation sont l'hématologie et la médecine interne $(67 \%=2 / 3$ et $4 / 6$ respectivement). On peut noter un pourcentage de succès faible de $27 \%(=3 / 11)$ pour les internes en néphrologie, alors que le pourcentage de projets de néphrologie acceptés parmi l'ensemble des projets acceptés est dans la moyenne des autres spécialités $(5,9 \%)$.

Notre étude ne nous permet pas de connaitre les causes de ces variations dans l'attribution des bourses en fonction des spécialités, même si certaines explications peuvent être évoquées. Les spécialités avec de nombreuses candidatures ont tendance à avoir un taux d'attribution plus faible car la commission préfère assurer la répartition des bourses dans l'ensemble des spécialités, plutôt que de privilégier la spécialité avec le plus de candidatures. En effet lorsque l'on regarde la répartition des financements « année-recherche » en fonction des spécialités, on constate que si le nombre de candidats par spécialité est très variable, la répartition est assez homogène parmi les différentes spécialités (tableau I). Sur cinq ans, les spécialités ayant obtenu le plus de financements « année-recherche » (pourcentage des projets retenus d'une spécialité par rapport à l'ensemble des projets retenus) sont la chirurgie générale (12\%), la neurologie et l'oncologie médicale $(9 \%)$, et celle ayant obtenu le moins de financement est la santé publique (2\%) (tableau I). Par ailleurs, dans certaines spécialités, un tuteur présente plusieurs candidats. La commission ne finançant en général qu'un projet par tuteur pour garantir que l'étudiant soit bien encadré, ces spécialités ont donc un taux d'attribution plus faible.

\section{Données provenant du questionnaire adressé aux internes}

Soixante-et-onze internes sur 112 ont répondu au questionnaire. Les données présentées dans la suite de cette lettre correspondent aux résultats issus des réponses au questionnaire. Soixante trois pour cent des internes, dont $46 \%$ de femmes, ont répondu au questionnaire. L'âge moyen de réalisation de l' « année-recherche » est de 27 ans et 6 mois ; $72 \%$ des candidats affichent une ambition hospitalouniversitaire.

La plupart des projets ont un laboratoire lyonnais comme lieu d'accueil (77\%), mais $13 \%$ des candidats désirent réaliser leur projet dans le reste de la France et $10 \%$ à l'étranger. Les projets se répartissent à part égale entre la recherche fondamentale $(39,5 \%)$ et translationnelle $(39,5 \%)$, avec un pourcentage moins important de projets purement cliniques 
Tableau I. Répartition des projets soumis et pourcentage de succès par diplôme d'études spécialisées (DES).

\begin{tabular}{|c|c|c|c|}
\hline D.E.S. & $\begin{array}{c}\text { Nombre } \\
\text { de candidats }\end{array}$ & $\begin{array}{c}\text { Pourcentage } \\
\text { de succès }(\%)^{2}\end{array}$ & Répartition $(\%)^{3}$ \\
\hline Anatomie et cytologie pathologiques & 3 & 67 & 3,9 \\
\hline Anesthésie-Réanimation & 9 & 22 & 3,9 \\
\hline Biologie Médicale & 5 & 0 & 0 \\
\hline Cardiologie et Maladies Vasculaires & 5 & 40 & 3,9 \\
\hline Chirurgie Générale & 15 & 40 & 11,8 \\
\hline Dermatologie et Vénérologie & 2 & 0 & 0 \\
\hline Endocrinologie et Métabolisme & 5 & 60 & 5,9 \\
\hline Gastro-entérologie et Hépatologie & 2 & 0 & 0 \\
\hline Gynécologie médicale & 1 & 0 & 0 \\
\hline Gynécologie-Obstétrique & 5 & 60 & 5,9 \\
\hline Hématologie & 3 & 67 & 3,9 \\
\hline Médecine Générale & 4 & 25 & 2,0 \\
\hline Médecine Interne & 6 & 67 & 7,8 \\
\hline Médecine Physique et de Réadaptation & 2 & 100 & 3,9 \\
\hline Néphrologie & 11 & 27 & 5,9 \\
\hline Neurochirurgie & 3 & 0 & 0 \\
\hline Neurologie & 9 & 56 & 9,8 \\
\hline Oncologie & 9 & 56 & 9,8 \\
\hline Ophtalmologie & 3 & 67 & 3,9 \\
\hline Pédiatrie & 6 & 50 & 5,9 \\
\hline Pneumologie & 3 & 100 & 5,9 \\
\hline Radiodiagnostic et Imagerie Médicale & 3 & 67 & 3,9 \\
\hline Rhumatologie & 2 & 0 & 0 \\
\hline Santé Publique & 3 & 33 & 2,0 \\
\hline
\end{tabular}

${ }^{1}$ Nombre de candidats par DES sur la période analysée (2010-2014).

2 Pourcentage de succès par DES, soit nombre de candidatures acceptées sur nombre de candidatures soumises pour chaque spécialité.

${ }^{3}$ La répartition correspond au pourcentage de projets accepté par DES sur l'ensemble des projets acceptés.

(10\%). Le projet est piloté dans $75 \%$ des cas par un titulaire hospitalo-universitaire.

Parmi les étudiants qui ont répondu au questionnaire, seuls $4 \%$ n'ont pas pu réaliser leur projet de recherche. Peu d'étudiants non sélectionnés ont déposé une nouvelle candidature au financement « annéerecherche $\gg(6 \%)$.

Un autre aspect important de cette enquête concerne le financement à proprement parler. La rémunération d'un interne en « année-recherche » est de 1634 euros nets par mois. Trente huit pour cent des internes ont rapporté le recours à des compléments de financement à type de garde ou de remplacement ; $18 \%$ des internes ayant répondu au questionnaire (succès ou échec au financement « année-recherche ») ont eu besoin de recourir à des fonds personnels pour réaliser leur année de recherche (pour couvrir en totalité ou en partie les frais liés à cette activité). Enfin, $38 \%$ des internes ont également rapporté l'obtention d'autres financements de recherche (bourses, subventions, prix) venant remplacer le financement « année recherche » ou en complément de celui-ci. Parmi les candidats non retenus $(32 / 69=46 \%), 91 \%$ d'entres eux ont pu réaliser leurs projets de recherches. Le 
financement a été assuré par d'autres fonds de recherche $(64 \%)$, des compléments de salaire par garde ou remplacement $(24 \%)$ ou le recours à des fonds propres $(27 \%)$.

L'expérience subjective des participants de cette enquête sur leur année de recherche est positive, avec une appréciation globale notée en moyenne à 7,5/10 . Il est à relever un taux très satisfaisant de production scientifique chez des étudiants qui réalisent pour la plupart un master 2 de recherche car $48 \%$ des participants ont pu écrire une publication et/ou participer à une conférence pour présenter leurs résultats. La réalisation d'une année de recherche semble remplir son objectif d'initiation à la recherche et de création de vocation de médecins chercheurs car $72 \%$ des participants témoignent d'une volonté de poursuivre leurs activités de recherche après leur " annéerecherche » et $52 \%$ d'entre eux ont déjà trouvé un laboratoire d'accueil ; $73 \%$ rapportent le souhait d'avoir un temps dédié à la recherche dans leur emploi du temps d'interne.

\section{Conclusions et perspectives}

Au total, cette enquête indique que le dispositif « année-recherche » semble remplir ses objectifs au sein du CHU de Lyon, avec un taux de réussite moyen sur la période analysée de $41 \%$. Si seulement $4 \%$ des internes ont dû abandonner leur projet, $18 \%$ des internes ont été obligés de le financer partiellement sur fonds propres. Par ailleurs, comme nous avons un pourcentage de réponse à notre questionnaire de $63 \%$, il est possible que nous sous-estimions le pourcentage d'internes qui ont dû abandonner leur projet. Ces résultats nous incitent donc, en période de réduction des dépenses de l'Assurance Maladie, à insister sur la nécessité de maintenir ce financement et d'augmenter, parallèlement au nombre d'internes, le nombre de postes alloués. En ce qui concerne les compléments de rémunération à type de garde ou de remplacement, bien que l'on puisse y voir une poursuite des activités cliniques au cours de cette année, il est évident que le temps passé à l'hôpital ou en clinique, les repos de sécurité et la fatigue engendrée nuisent à l'activité de recherche.
Par ailleurs, $10 \%$ des internes souhaitent réaliser des projets à l'étranger, ce qui implique des coûts supplémentaires. Ces projets sont primordiaux car ils permettent d'établir des collaborations scientifiques internationales et ils favorisent le rayonnement de la recherche française. Il serait intéressant de mettre en place un dispositif pour valoriser le financement de ce type de projet.

D'après notre étude, la réalisation d'une « annéerecherche » est utile pour développer des vocations pour la recherche car à l'issue de cette année $72 \%$ des internes souhaitent poursuivre une activité de recherche. Il serait intéressant de voir sur le long terme le pourcentage d'internes qui pourront, effectivement, garder leur double valence de chercheur et de clinicien.

Benjamin J. BLAISE ${ }^{1}$, Martine LAVILLE ${ }^{1,2}$, Françoise BORSON-CHAZOT ${ }^{1,2}$, Jean-François NICOLAS ${ }^{1,3}$ et Amélie BOESPFLUG ${ }^{1}$

${ }^{1}$ Hospices Civils de Lyon, Lyon, France

${ }^{2}$ Université de Lyon, Université Claude Bernard Lyon 1, Faculté de Médecine Lyon-Est, Lyon, France

${ }^{3}$ Université de Lyon, Université Claude Bernard Lyon 1, Faculté de Médecine et de Maïeutique Lyon-Sud, Pierre-Bénite, France

\section{Remerciements}

Les auteurs remercient le professeur J. Etienne pour sa relecture attentive.

\section{Référence}

1. Arrêté du 21 janvier 2016 modifiant l'arrêté du 4 octobre 2006 modifié définissant les modalités d'organisation de l'année-recherche durant le troisième cycle des études de médecine, d'odontologie et de pharmacie. JORF n ${ }^{\circ} 0024$ du 29 janvier 2016. 2016 [On-line] Disponible sur : https://www.legifrance.gouv.fr/eli/arrete/2016/1/21/ AFSH1602096A/jo

Correspondance et offprints : Benjamin Blaise.

Mailto : benjamin.blaise@chu-lyon.fr 